\title{
Detecting disulfide crosslinks of high-molecular weight complexes in mouse SVS proteins by diagonal electrophoresis
}

\author{
Han-Jia Lin ${ }^{\text {a }}$, Chun-Hui Lin ${ }^{b}$, Huan-Chin Tseng ${ }^{b}$, Yee-Hsiung Chen ${ }^{\text {a,b,* }}$ \\ ${ }^{a}$ Institute of Biological Chemistry, Academia Sinica, National Taiwan University, Taipei, Taiwan \\ ${ }^{\mathrm{b}}$ Institute of Biochemical Sciences, College of Life Science, National Taiwan University, Taipei, Taiwan \\ Received 9 November 2005 \\ Available online 20 March 2006
}

Since Brown and Hartley first developed two-dimensional electrophoresis on a paper sheet to examine the interpolypeptide disulfide linkage of chymotrypsinogen $\mathrm{A}$ in 1966 [1], diagonal electrophoresis has become one of the most useful methods for the protein study in this regard. In 1974, Sommer and Traut extended the concept to diagonal polyacrylamide-dodecyl sulfate electrophoresis for identification of ribosomal proteins crosslinked with methyl-4mercaptobutyrimidate [2], with their protocol being generally followed since that time. Briefly, one strip of gel containing the protein sample is cut from the gel slab of first-dimensional electrophoresis being conducted under a nonreducing condition. After treatment with a disulfidescissoring reagent, the gel is embedded into another polyacrylamide gel slab for the second-dimensional electrophoresis under a reducing condition. The resultant protein samples containing no interpolypeptide disulfide bond(s) will lie in a diagonal line on the second-dimensional gel slab, whereas a reduced sample will be resolved into smaller protein components. Given the various forms of highmolecular protein complexes (HMPCs) ${ }^{1}$ in mouse seminal vesicle secretion (SVS) [3], we attempted to apply this conventional method to study the detail of interpolypeptide disulfide crosslinks of each HMPC. We discovered, however, that clear identification of HMPC from the protein spots on the gel slab of second-dimensional electrophoresis is somewhat problematic. In their study of rat SVS proteins in 1987, Wagner and Kistler reported similarly [4]. In this article, we present a modified method that improves the effectiveness of the established version.

\footnotetext{
* Corresponding author. Fax: +886223635038.

E-mail address: bc304@gate.sinica.edu.tw (Y.-H. Chen).

1 Abbreviations used: HMPC, high-molecular protein complex; SVS, seminal vesicle secretion; DTT, dithiothreitol.
}

Mouse SVS was collected from outbred ICR mice according to the established procedure [5-7]. The method of Laemmli was adopted to prepare the protein samples for SDS-PAGE [8]. Approximately $20 \mu \mathrm{g}$ of SVS was dissolved in a sample buffer containing $2 \%$ SDS, $10 \%$ glycerol, $0.005 \%$ bromophenol blue, and $100 \mathrm{mM}$ dithiothreitol (DTT) in $50 \mathrm{mM}$ Tris- $\mathrm{HCl}$ at $\mathrm{pH}$ 6.8. The sample solution was heated at $95^{\circ} \mathrm{C}$ for $5 \mathrm{~min}$ before loading for SDS-PAGE. According to the previous study [7], the major protein components in mouse SVS were designated SVS I ( $92 \mathrm{kDa})$, SVS II ( $38 \mathrm{kDa})$, SVS III ( $34 \mathrm{kDa})$, SVS IV $(15 \mathrm{kDa})$, and SVS V $(14 \mathrm{kDa})$, as well as a minor (and then unreported) protein component SVSP55 $(55 \mathrm{kDa})$, based on their mobility of SDS-PAGE on a $14 \%$ gel slab under a reducing condition (Fig. 1A, lane 2). Meanwhile, SDS-PAGE was performed for the protein sample prepared in the sample buffer devoid of DTT on a $6 \%$ polyacrylamide slab under nonreducing conditions to improve resolution of the HMPC variants in mouse SVS. We found that distortion of the protein bands on the soft gel often occurred, in particular bending around their horizontal edge. This resulted in two bothersome disadvantages. First, further resolution of such distorted bands by second-dimensional electrophoresis produced tailing spots on the gel slab. This was problematic in terms of the assignment of HMPC components. Second, there were technical difficulties not only in cutting the protein-containing strip from the first-dimensional soft gel slab but also in inserting such a soft strip into the chamber for second-dimensional electrophoresis.

The above shortcomings can be rectified when the firstdimensional electrophoresis is performed on a gel slab prepared in an apparatus constructed using two plain glass plates, one of which is attached to a Gel Bond PAG film (Cambrex Bio Science, Rockland, ME, USA), which serves as a gel supporter. It was found that gels prepared from acrylamide concentrations as low as $4 \%$ still strongly adhere 
A

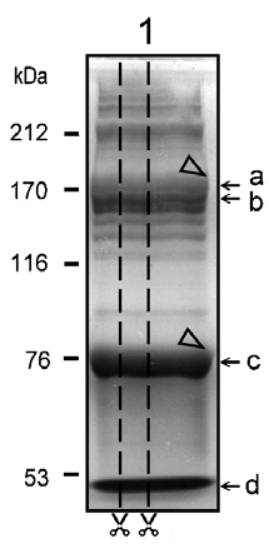

B

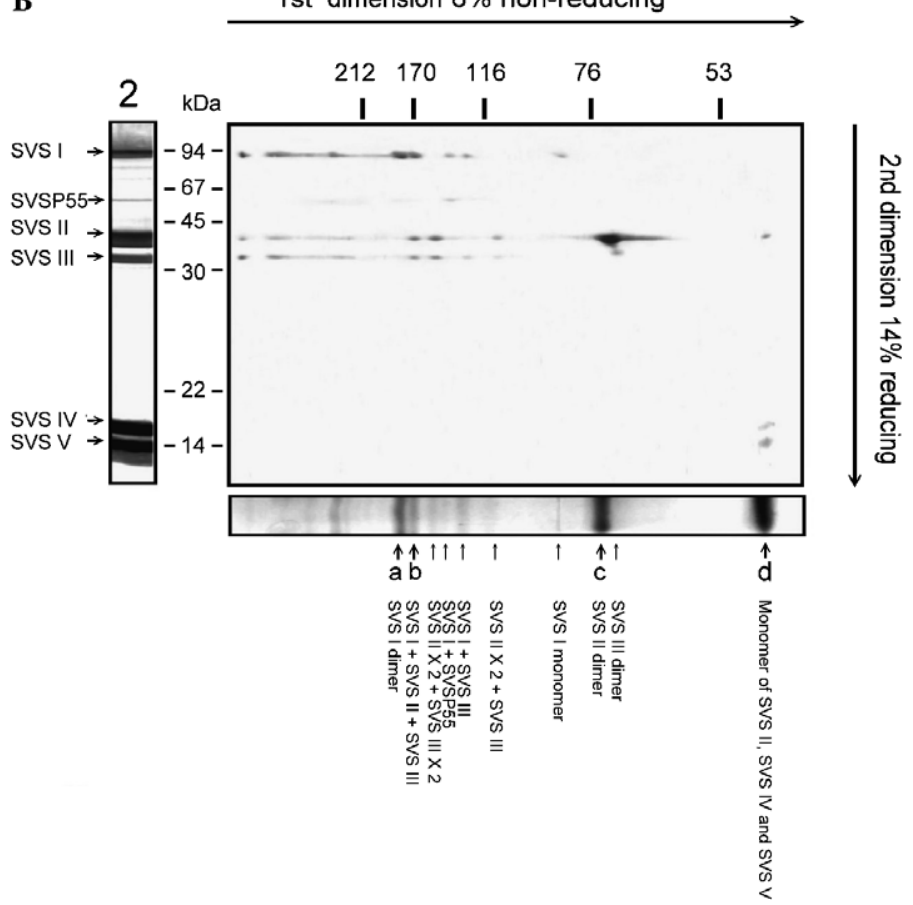

Fig. 1. Easy manipulation and high-resolution diagonal electrophoresis. (A) The protein components of mouse SVS (20 $\mu \mathrm{g})$ were resolved by SDS-PAGE on a $14 \%$ gel slab under reducing conditions (lane 2). Alternatively, the electrophoresis was conducted on a Gel Bond film-supporting $6 \%$ gel slab under nonreducing conditions (lane 1). The film-supporting gel was stained and dried as described in the text. The arced edge and tailing of a protein band are indicated by open arrowheads. A strip of gel containing the distortion-free protein bands was scissored from the film-supporting gel slab (dashed lines of lane 1) for the second-dimensional electrophoresis, which was performed on $14 \%$ SDS-PAGE $(8 \times 10 \mathrm{~cm})$ under reducing conditions (B). The major HMPC bands on the first dimension are denoted a-d, and the composition of each nonreducing protein band, denoted on the bottom of the first-dimensional gel, is revealed from the silver-stained protein spots on the two-dimensional gel pattern.

to the film. The electrophoresis of the SVS proteins was conducted on a film-supporting $6 \%$ polyacrylamide slab without disulfide reduction at $15 \mathrm{~V} / \mathrm{cm}$ for $60 \mathrm{~min}$. Without prefixation of the protein bands and after electrophoresis, the film adherent gel was directly washed twice with distilled water for 10 min, followed by staining with GelCode Blue reagent (Pierce, IL, USA) to reveal the protein band. As shown in lane 1 of Fig. 1A, the various forms of HMPC were well resolved. Four major bands emerged from the first-dimensional electrophoresis: a $(184 \mathrm{kDa}), \mathrm{b}(164 \mathrm{kDa}), \mathrm{c}(76 \mathrm{kDa})$, and $\mathrm{d}(<53 \mathrm{kDa}$, appearing around the button of the gel slab). Minor bands bearing $M_{\mathrm{r}}$ values greater than $76 \mathrm{kDa}$ also emerged. The appearance of various nonreduced protein bands that have $M_{\mathrm{r}}$ values greater than any of the reduced protein components manifests the complication of intermolecular disulfide bridges among the SVS protein components. Before the second-dimensional electrophoresis, the gel was dried overnight on the film at $37^{\circ} \mathrm{C}$ after soaking in $2 \%$ glycerol for $1 \mathrm{~h}$. This provides three technical advantages over the conventional second-dimensional method. First, the dried gel can be stored for more than 1 month without distortion of the protein bands. Second, the deformed portion around the horizontal edges of the stained protein bands could be scissored out easily before the second-dimensional electrophoresis (see lane 1 of Fig. 1A). Third, rehydration of the dried gel and the reduction reaction for the protein bands could proceed simultaneously. For the disulfide reduction, the dried gel was soaked in a reaction buffer containing $6 \mathrm{M}$ urea, $20 \%$ glycerol, $2 \%$ SDS, $0.005 \%$ bromophenol blue, and $100 \mathrm{mM}$ DTT in $50 \mathrm{mM}$ Tris- $\mathrm{HCl}(\mathrm{pH} 6.8)$ for $1 \mathrm{~h}$ at room temperature. After the reduction, the gel was incubated in the reaction buffer, in which DTT was replaced by $135 \mathrm{mM}$ iodoacetamide to block the thio group. This treatment does not cause detachment of the polyacrylamide gel from the supporting film, facilitating its insertion into the chamber for second-dimensional electrophoresis.

The protein bands on the film-supporting gel were reduced as outlined above, and their components were resolved by second-dimensional electrophoresis on a $14 \%$ polyacrylamide slab under reducing conditions. After the gel slab was stained with silver nitrate, the protein spots on the two-dimensional gel pattern were clearly revealed (Fig. 1B). The unequivocal assignment of molecular size for the protein spots enabled confident elucidation of the interpolypeptide disulfide crosslinks in each HMPC, as shown at the bottom of Fig. 1B. There are no cysteine residues in SVS IV and SVS $\mathrm{V}$, there is one cysteine residue in SVS II, there are three cysteine residues in SVS III, and there are six cysteine residues in SVS I. Band d contains mainly SVS II, SVS IV, and SVS V, suggesting no disulfide crosslinking among these three proteins. The interpolypeptide disulfide crosslinking among SVS I, SVS II, and SVS III may produce various forms of protein complexes. This is demonstrated by the presence of the homo-polypeptide dimers of SVS I (band a), SVS II (band c), 
and SVS III; the hetero-polypeptide complexes formed by two SVS II plus one SVS III, one SVS I plus one SVS III, two SVS II plus two SVS III, one SVS I plus one SVS II plus SVS III (band b), one SVS I plus one SVSP55; and the even larger complexes with $M_{\mathrm{r}}$ values greater than $212 \mathrm{kDa}$ formed by unknown stoichiometric ratios of SVS I, SVS II, and SVS III. These data provide a sound basis for future study to identify the roles of polypeptide complexes in semen coagulation that are important for the reproduction of a substantiated number of mammalian species [3].

In summary, the protocol reported here facilitates physical manipulation of a single soft gel prepared from a low concentration of acrylamide, providing good-quality identification of the high-molecular weight complexes formed by disulfide bridges. Our work may help to improve the reliability and applicability of diagonal electrophoresis.

\section{Acknowledgments}

This work was supported in part by Grants NSC942311-B-002-004 and NSC94-2311-B-001-016 from the National Sciences Council (Taiwan).

\section{References}

[1] J.R. Brown, B.S. Hartley, Location of disulphide bridges by diagonal paper electrophoresis: the disulphide bridges of bovine chymotrypsinogen A, Biochem. J. 101 (1966) 214-228.

[2] A. Sommer, R.R. Traut, Diagonal polyacrylamide-dodecyl sulfate gel electrophoresis for the identification of ribosomal proteins crosslinked with methyl-4-mercaptobutyrimidate, Proc. Natl. Acad. Sci. USA 71 (1974) 3946-3950.

[3] H.G. Williams-Ashman, Transglutaminases and the clotting of mammalian seminal fluids, Mol. Cell Biochem. 58 (1984) 51-61.

[4] C.L. Wagner, W.S. Kistler, Analysis of the major large polypeptides of rat seminal vesicle secretion: SVS I, II, and III, Biol. Reprod. 36 (1987) $501-510$.

[5] H.J. Lin, C.W. Luo, Y.H. Chen, Localization of the transglutaminase cross-linking site in SVS III, a novel glycoprotein secreted from mouse seminal vesicle, J. Biol. Chem. 277 (2002) 3632-3639.

[6] C.W. Luo, H.J. Lin, Y.H. Chen, A novel heat-labile phospholipid-binding protein, SVS VII, in mouse seminal vesicle as a sperm motility enhancer, J. Biol. Chem. 276 (2001) 6913-6921.

[7] Y.H. Chen, B.T. Pentecost, J.A. McLachlan, C.T. Teng, The androgendependent mouse seminal vesicle secretory protein IV: characterization and complementary deoxyribonucleic acid cloning, Mol. Endocrinol. 1 (1987) 707-716.

[8] U.K. Laemmli, Cleavage of structural proteins during the assembly of the head of bacteriophage T4, Nature 227 (1970) 680-685. 